\title{
Suppression of Ground-State Magnetization in Finite-Sized Systems Due to Off-Diagonal Interaction Fluctuations
}

\author{
Philippe Jacquod and A. Douglas Stone \\ Department of Applied Physics, P. O. Box 208284, Yale University, New Haven, CT 06520-8284
}

(August 8, 2018)

\begin{abstract}
We study a generic model of interacting fermions in a finite-sized disordered system. We show that the off-diagonal interaction matrix elements induce density of states fluctuations which generically favor a minimum spin ground state at large interaction amplitude, $U$. This effect competes with the exchange effect which favors large magnetization at large $U$, and it suppresses this exchange magnetization in a large parameter range. When off-diagonal fluctuations dominate, the model predicts a spin gap which is larger for odd-spin ground states as for even-spin, suggesting a simple experimental signature of this off-diagonal effect in Coulomb blockade transport measurements.

PACS numbers : 73.23.-b, 71.10.-w, 71.24.+q, 75.10.Lp
\end{abstract}

Ferromagnetic instabilities result from the combined effect of the electronic interactions together with the Pauli principle. The interaction energy can be minimized when the fermionic antisymmetry requirement is satisfied by the spatial wavefunction leading to alignment of spins and a large ground-state spin magnetization (a familiar example of this is Hund's rule in atoms). In contrast when the interaction is weak minimal spin is favored because it costs kinetic energy to flip a spin as it must then be promoted to a higher energy level. When treating ferromagnetism in metals, because of the locality of the Pauli principle, magnetic instabilities are usually studied in the framework of the Hubbard model taking only the short-range part of electronic interactions into account, so that only pairs of electrons of opposite spin interact. As the magnetization increases the number of interacting pairs decreases and the system spontaneously magnetizes for sufficiently strong interactions. In a finite-sized system this Stoner instability [1] occurs when the typical exchange interaction between two states close to the Fermi energy is equal to the one-particle level spacing which for a clean system with Hubbard interaction gives $U_{c}=\Delta$.

There has been much recent interest in the Stoner instability of finite-size disordered metals such as quantum dots and metallic nanoparticles [2]. Building on earlier perturbative work [3] Andreev and Kamenev [4] recently found a significant reduction of the Stoner threshold in disordered systems due to spatial correlations in diffusive wavefunctions which enhance the average exchange term. More recently, Brouwer et. al. 5] considered the effect of mesoscopic wavefunction fluctuations and found an associated increase of the probability of non-zero ground state spin magnetization below the Stoner threshold. Within a similar model, Baranger et. al. [6] proposed that spontanous magnetization effects could explain kinks in the field-dependence of Coulomb blockade resonances. The purpose of the present paper is to point out a competing effect of interactions which suppresses the probability of ground-state magnetization and has not been treated in any of the previous works on itinerant magnetism of disordered systems. The mean-field treatments lead- ing to an exchange term in the effective hamiltonian for disordered metals neglects the effects of off-diagonal interaction matrix elements 四; however it is well-known from studies of nuclei and atoms [7] that the band-width of the many-body density of states in finite interacting fermi systems is actually determined by the fluctuations of these off-diagonal matrix elements. When one introduces the spin degree of freedom into these models we shall see below that one immediately finds that these fluctuations are largest for the states of minimal spin. This effect then strongly increases the probability that the extremal (low-lying) states in the band are those of minimal spin and opposes the exchange effect. We expect this effect to be significant in quantum dots and to suppress the possibility of high spin ground states.

We start from the Hamiltonian for $n$ spin- $1 / 2$ particles

$$
H=\sum \epsilon_{\alpha} c_{\alpha, s}^{\dagger} c_{\alpha, s}+\sum U_{\alpha, \beta}^{\gamma, \delta} c_{\alpha, s}^{\dagger} c_{\beta, s^{\prime}}^{\dagger} c_{\delta, s^{\prime}} c_{\gamma, s}
$$

$s^{\left({ }^{\prime}\right)}=\uparrow, \downarrow$ are spin indices. The $m / 2$ different one-body energies are distributed as $\epsilon_{\alpha} \in[-m / 2 ; m / 2]$ so as to fix $\Delta \equiv 1$ with spin degeneracy. The interaction commutes with the $z$-component $\sigma_{z}$ of the total magnetization $\sigma$ so that the Hamiltonian acquires a block structure where blocks are labelled by $\sigma_{z}$ and due to Spin Rotational Symmetry (SRS) subblocks of given $\sigma \geq\left|\sigma_{z}\right|$ appear within each of these blocks. Each block's size is given in term of binomial coefficients as $N\left(\sigma_{z}\right)=\left(\begin{array}{l}m / 2 \\ n / 2-\sigma_{z}\end{array}\right)\left(\begin{array}{l}m / 2 \\ n / 2+\sigma_{z}\end{array}\right)$.

The Hamiltonian (1) can be viewed as a generic model of interacting fermions expressed in the basis of Slater determinants constructed from the eigenstates $\psi_{\alpha}$ of the corresponding free fermions model $H_{0} \equiv \sum \epsilon_{\alpha} c_{\alpha, s}^{\dagger} c_{\alpha, s}$. In this basis the interaction matrix elements are given by $U_{\alpha, \beta}^{\gamma, \delta}=\int d \vec{r} d \overrightarrow{r^{\prime}} U\left(\vec{r}-\overrightarrow{r^{\prime}}\right) \psi_{\alpha}^{*}(\vec{r}) \psi_{\beta}^{*}\left(\overrightarrow{r^{\prime}}\right) \psi_{\gamma}(\vec{r}) \psi_{\delta}\left(\overrightarrow{r^{\prime}}\right)$, where $U\left(\vec{r}-\overrightarrow{r^{\prime}}\right)$ is the interaction potential. Due to disorder or chaotic boundary scattering the wavefunctions $\psi_{\alpha}$ have a random character leading to fluctuations in $U_{\alpha, \beta}^{\gamma, \delta}$ around their average value. We take these fluctuations to be random with a zero-centered gaussian distribution of width $U$. This gives a contribution $\bar{H}$ similar to the 
second term in the right-hand side of (11) with a distribution $P\left(U_{\alpha, \beta}^{\gamma, \delta}\right) \propto e^{-\left(U_{\alpha, \beta}^{\gamma, \delta}\right)^{2} / 2 U^{2}}$ of interaction matrix elements. Only diagonal matrix elements $U_{\alpha, \beta}^{\alpha, \beta}$ and $U_{\alpha, \beta}^{\beta, \alpha}$ have a nonzero average leading to mean-field chargecharge and spin-spin diagonal interactions 䐗. We neglect the charge-charge contribution as it has no influence on the magnetization and this leaves us with the following effective Hamiltonian

$$
\mathcal{H}=H_{0}+\bar{H}-\lambda U \sum \vec{s}_{\alpha} \vec{s}_{\beta}
$$

$\vec{s}_{\alpha} \equiv \sum_{s, t} c_{\alpha, s}^{\dagger} \vec{\sigma}_{s, t} c_{\alpha, t}$ are spin operators and the ferromagnetic spin-spin interaction has a strength $\lambda U>0$. Without it $(\lambda=0)$, the Hamiltonian (2) within each spin block is precisely the Two-Body Random Interaction Model (TBRIM) introduced in nuclear physics [8], and used to study thermalization [9] and the emergence of quantum chaos in few-body systems [10] and statistical features observed in shell model calculations [11,12]. A similar model has been shown recently to be consistent with the observed gaussian distribution of peak-spacings in Coulomb blockade resonances through quantum dots [13. One key feature of the TBRIM is that the manybody density of states (MBDOS) has an approximately gaussian shape with a variance proportional to the connectivity $K$, i.e. the number of non-zero matrix elements in each row [8]. Similarly we can estimate the variance of the MBDOS of the Hamiltonian (2) for fixed $\left(\sigma, \sigma_{z}\right)$ and $U / \Delta \gg 1$ as

$$
\frac{1}{N\left(\sigma_{z}\right)} \sum_{I, J} \bar{H}_{I, J}^{2} \delta\left(\sigma_{z}^{(I)}-\sigma_{z}\right) \delta\left(\sigma^{(I)}-\sigma\right) \approx K U^{2}
$$

where $\bar{H}_{I, J}=\langle I|\bar{H}| J\rangle$ and $|I\rangle$ refers to a Slater determinant. Hence each block's bandwidth goes as $\sqrt{K} U$ with a $\sigma_{z}$-dependent connectivity which can be expressed as $K\left(n, m, \sigma_{z}\right) \approx 1+C\left(n / 2+\sigma_{z}, m / 2\right)+C\left(n / 2-\sigma_{z}, m / 2\right)+$ $1 / 2\left((n / 2)^{2}-\sigma_{z}^{2}\right)\left((m / 2-n / 2)^{2}-\sigma_{z}^{2}\right)$ in term of the function $C(n, m)=n(m-n)+n(n-1)(m-n)(m-n-1) / 4$. The factor $1 / 2$ in front of the last contribution to $K$ is needed to take into account the effect of SRS. In the dilute limit approximately half of the spin-flip transitions which conserve $\sigma_{z}$ are not allowed because they don't conserve total spin (e.g. change a singlet to triplet). The estimate (3) assumes that each matrix element has the same variance, which for a generic off-diagonal element is $\sim U^{2}$. However it is easily seen that diagonal matrix elements $\bar{H}_{I, I}$ have an enhanced variance $\sim\left(3 n^{2} / 4+\sigma_{z}^{2}\right)^{2} U^{2}$ which induces deviations from (3) for large filling and large magnetization. Nevertheless, these matrix elements can be neglected in the dilute and weakly-magnetized limit $1 \ll n / 2+\sigma_{z} \ll m$ where the larger number of offdiagonal matrix elements dominates the variance when $(m-n)^{2} \gg\left(3 n^{2} / 4+\sigma_{z}^{2}\right)$. In the right inset to Fig. 1 we show plots of $K\left(n, m, \sigma_{z}\right)$ for different filling factors $\nu=n / m$ as well as a comparison with the true variance of the MBDOS for $\nu=3 / 8$. Deviations from the estimate (3) are small, even at this rather large filling and increase with increasing magnetization in agreement with the above reasoning. Moreover in the left inset to Fig. 1 we show that the full MBDOS follows the scaling (3) with significant deviations only at large magnetization, so that it is plausible that this scaling also determines the tails in which the ground state energies will be found.

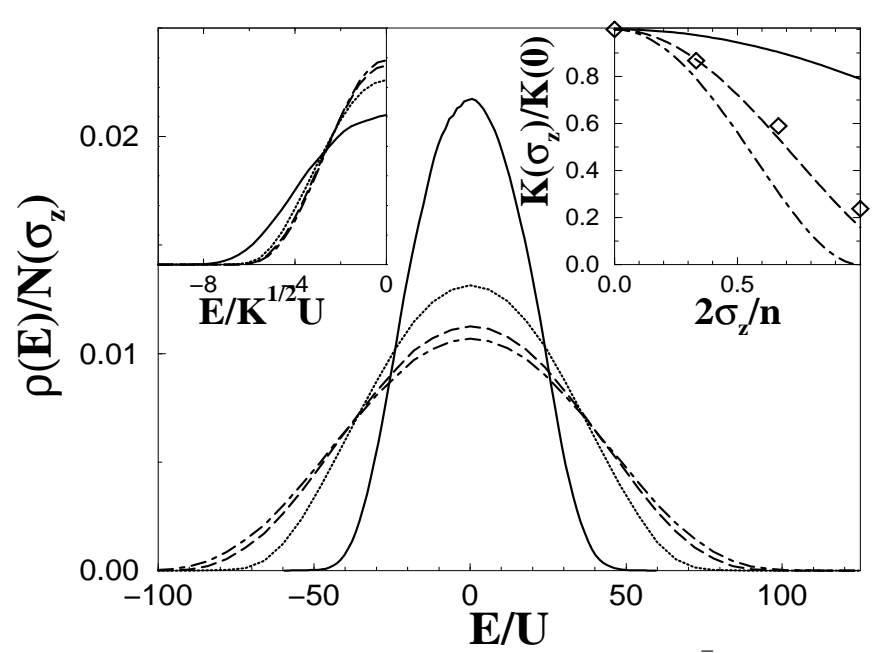

FIG. 1. Density of states for the Hamiltonian $\bar{H}$ with $n=6$ particles and $m=16$ orbitals, corresponding to the magnetization blocks $\sigma_{z}=-3$ (solid line), -2 (dotted line), -1 (dashed line) and 0 (dotted-dashed line). Left inset: rescaled density of states showing the approximate scaling in $E / K^{1 / 2} U$. Right inset : Normalized connectivity $K\left(\sigma_{z}\right) / K(0)$ vs. magnetization for filling factors $\nu=1 / 10$ (solid line), $3 / 8$ (dashed line) and $1 / 2$ (dotted-dashed line). We compare this estimate to the true variance for $\nu=3 / 8$ obtained numerically from the data of the main figure (diamonds).

Our essential finding follows from the simple features of the model already stated. The full MBDOS is a sum of approximately gaussian contributions from each spin block with a variance proportional to the corresponding connectivity. The latter is a monotonously decreasing function of $\sigma_{z}$. Hence the broadest MBDOS corresponds to the minimally magnetized block and the ground state will be found in this block with increased probability [14]. Assuming, as just discussed, that the tails of the distribution scale with the variance with a factor $\beta$ and neglecting contributions arising from $H_{0}$, the typical spin gap can be estimated (for $\lambda=0$ ) as

$$
\Delta_{s}^{U} \approx \beta U\left[\sqrt{K\left(\left|\sigma_{\min }\right|\right)}-\sqrt{K\left(\left|\sigma_{\min }\right|+1\right)}\right]
$$

This multiple gaussian structure of the MBDOS and the scaling with $\sqrt{K}$ obtained from numerical calculations are shown in Fig. 1 for $\lambda=0$. For $\lambda \neq 0$, the spin-spin interaction induces relative shifts of each block's MBDOS which eventually will shift the finite spin blocks sufficiently to overcome the larger fluctuations of the minimal 
spin MBDOS. This is the competition between exchange and off-diagonal fluctuations already mentioned. However for reasonable values of $\lambda$ the off-diagonal fluctuations strongly reduce the probability of exchange-induced magnetization (see Fig. 3).

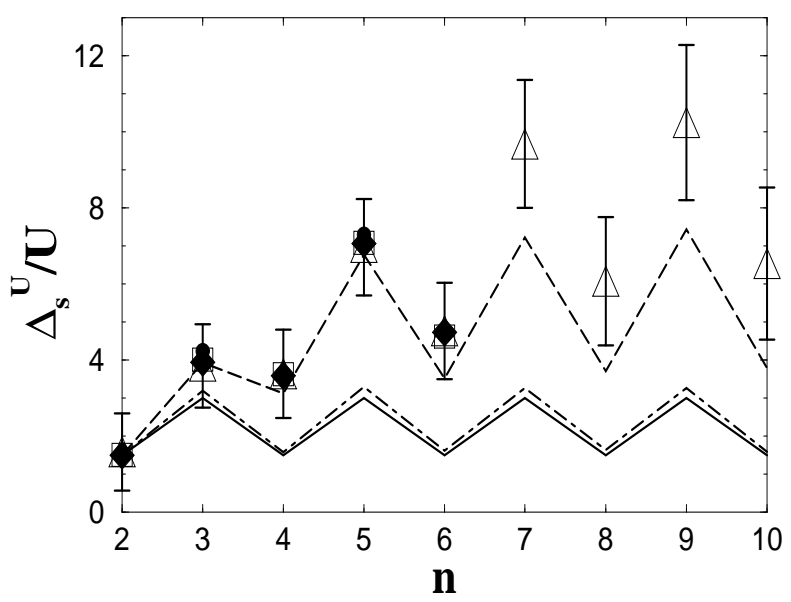

FIG. 2. Dependence of the finite-size spin gap in the number $n$ of particles. Points correspond to numerical results for $m=10$ (full circles), 12 (empty squares), 14 (full diamonds) and 16 (empty triangles) and the solid line to the dilute estimate (4) with a numerical factor $\beta=1.5$. For the case $m=16$ and 1000 Hamiltonian realizations, the error bars indicate the r.m.s. of the gap distribution while the dashed and dotted-dashed lines show the numerically computed variances (Left-hand side of eq. (3)) for the full Hamiltonian and after setting to zero non-generic interaction matrix elements respectively.

In Fig. 2 we show the computed spin gap $\Delta_{s}^{U}$ between the minimally magnetized ground-state and the first spin excited level for $\lambda=0$ in the limit of dominant interaction, i.e. neglecting $H_{0}$ in (1). One of the main features is a strong even-odd effect which is reminiscent of a similar behavior in the limit of vanishing interactions. However the origin here is the fluctuating interaction and the energy differences scale as $U$ instead of $\Delta$. We next note that the gap first increases with increasing number of particles before it seems to stabilize above $n=6$. We have checked (dashed and dot-dashed lined in Fig. 2) that this behaviour, which is not captured by the dilute estimate (4), is partly due to the neglect in (3) of nongeneric matrix elements with enhanced variance mentioned above. However, even though the exact variance gives a much better estimate, it still underestimates the gap at larger $n$ and we have numerically determined that this is due to a strong positive correlation of the ground state energies in adjoining spin blocks $(\sim 0.9)$. Such correlations, although interesting, are not suprising since the different block hamiltonians are not statistically independent (many of the same two-body matrix elements appear in both).
We next switch on the mean-field spin-spin interaction $\lambda>0$ which induces energy shifts of $-\lambda U\left|\sigma_{z}\right|\left(\left|\sigma_{z}\right|+1\right)$ [14. On average the spin gap becomes $\Delta_{s}=\Delta_{s}^{U}-\bar{\lambda} U$, where $\bar{\lambda}=\left(5-(-1)^{n}\right) \lambda / 2$, i.e. the relative shift between the two lowest magnetized blocks is larger for odd number of particles. The variance of the gap distribution is unaffected and we can already conclude that the probability $P\left(\sigma_{z}>0\right)$ of finding a magnetized ground-state is reduced by the off-diagonal matrix elements and saturates above a finite value $U_{c}$, since the width of the gap distribution is proportional to its average $\sim U$. This is shown on Fig. 3 where $P\left(\sigma_{z}>0\right)$ is plotted against $U / \Delta$ for different values of $\lambda$. On the same graph we show numerical results obtained after setting to zero the off-diagonal matrix elements. The data unambiguously reflect the strong demagnetizing influence of the off-diagonal matrix elements.

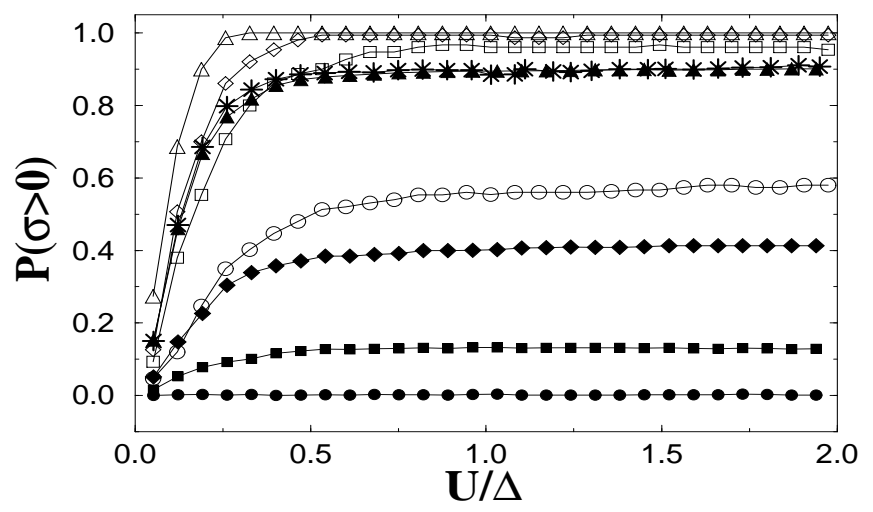

FIG. 3. Probability for a magnetized ground-state as a function of $U / \Delta$ for 2000 realizations of Hamiltonian (2) with $n=5$ and $m=10, \lambda=2,4,5$ and 7 (full symbols, from bottom to top). Empty symbols show the corresponding curves after setting to zero the off-diagonal matrix elements. Stars correspond to $n=5, m=14$ and $\lambda=7$.

The physically relevant value of $\lambda$ will depend on the microscopic details of the system. Indeed $\lambda$ is given by half the ratio of the mean exchange interaction with the fluctuations of off-diagonal matrix elements [4] $\lambda=\left\langle U_{\alpha, \beta}^{\beta, \alpha}\right\rangle / 2$ r.m.s. $\left(U_{\alpha, \beta}^{\gamma, \delta}\right)$. Semiconductor quantum dots with poor screening and extended, chaotic single-particle wavefunctions should have $\lambda \approx 1$ while for extended diffusive metallic systems, we get a much larger spin-spin interaction $\lambda \sim g$ where $g>1$ is the system's conductance [3]. From Fig. 3 however, we see that even in the regime of dominating interactions, a nonmagnetized ground state is more probable for $\lambda \ll 5\left(\bar{\lambda} U \ll \Delta_{s}^{U}\right)$.

We finally consider the influence of an external magnetic field which only introduces a Zeemann coupling. This situation can be experimentally realized by applying a magnetic field in the plane of a two-dimensional electron gas. This Zeemann term does not affect the Hamil- 
tonian's block structure, but only shifts each block's MBDOS by an amount $g \mu_{B} B \sigma_{z}$. Due to the spin gap discussed above, a finite magnetic field of average magnitude $\left\langle B_{c}\right\rangle=\Delta_{s} / g \mu_{B}$ is necessary to magnetize the system. The even-odd effect emphasized in Fig. 2 results in a critical field to flip one spin which is significantly larger for a $\sigma_{z}= \pm 1 / 2$ (odd) ground state as compared to a $\sigma_{z}=0$ (even) ground state. More generally the gap for a lower spin state is smaller than that for the state of higher spin (this follows from the right inset to Fig. 1). This aspect of our theory can be tested experimentally by studying the in-plane magnetic field dependence of the position of Coulomb blockade conductance peaks at very low temperature $T \ll \Delta$. The resonant gate voltage is given by a difference of two many-body ground-state energies $e V_{g}^{n}=E_{n+1}^{0}-E_{n}^{0}$, and it is always the difference of an even-odd pair. The peak position behaves like

$$
e V_{g}^{n}(B)=E_{n+1}^{0}-E_{n}^{0}+g \mu_{B} B \delta \sigma_{z}(n)
$$

where $\delta \sigma_{z}(n)$ is the magnetization difference between the two consecutive ground-states. Without magnetization $\delta \sigma_{z}(n)=(-1)^{n} / 2$ and one has $\left|\partial V_{g} / \partial B\right|=g \mu_{B} / 2$. As $B$ is increased the ground-state with even number of electrons is most likely to magnetize first, exactly reversing the slope of two consecutive peaks; then as the field increases further the odd state will likely flip, restoring the original slope. As long as consecutive ground states never differ by more than one unit of spin the absolute value of the slope will remain constant as the system polarizes.

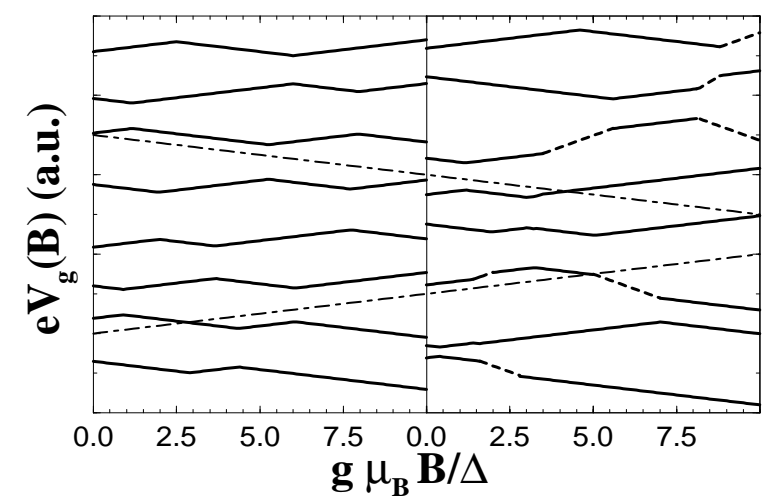

FIG. 4. Schematic of the conductance peaks in a 2D quantum dot as a function of an in-plane magnetic field for $m=14$, $\lambda=1$ (left) and 2.5 (right) and $U / \Delta=4$ corresponding to the addition of the $n=3,4, \ldots 10^{\text {th }}$ electrons (from bottom to top). Dotted-dashed lines indicate slopes of $\pm g \mu_{B} / 2$. Larger slopes for which spin-blockade effects strongly reduce the peak height [15] are indicated by dashed segments. Note that due to the subtraction of the average charge-charge interaction, the model does not reproduce the charging energy so that the vertical distance between consecutive peaks is arbitrary.

However if there exist many magnetized ground states then one expects a range of slopes to occur. In this case the corresponding peak heights will be strongly reduced by the spin blockade mechanism (see dashed lines on Fig. 4) [15] which should be easily visible experimentally. This argument neglects changes in the $g$-factor of the electron with changing $n$, which presumably are slow. The evenodd behavior of $\Delta_{s}$ is qualitatively similar to the noninteracting case, however the scale in $g \mu_{B} B$ over which spin flips occur is determined by $U$ and not $\Delta$. Typically, this results in an increase of the field necessary to achieve full polarization on the dot. This is illustrated on Fig. 4 where the peaks positions are drawn as a function of the Zeemann coupling for $\lambda=1.5$ and 3. It is clearly seen that at small $\lambda,\left|\partial V_{g} / \partial B\right|$ is constant and corresponds to a minimal $\delta \sigma_{z}$, while increasing $\lambda$ gives different slopes in agreement with the above reasoning.

Numerical computations were performed at the Swiss Center for Scientific Computing and at the National Energy Research Scientific Computing Center. Work supported by the NSF grant PHY9612200 and the Swiss National Science Foundation. We acknowledge helpful discussions with S. Åberg, Y. Alhassid, F. Izrailev, S. Tomsovic, M. Vojta and D. Weinmann.

[1] S. Doniach and E. Sondheimer "Green's Functions for Solid State Physicists", Addison-Wesley (1974).

[2] D. C. Ralph et al., Phys. Rev. Lett. 74, 3241 (1995); D. Davidović and M. Tinkham, Phys. Rev. Lett. 83, 1644 (1999).

[3] B.L. Altshuler and A.G. Aronov in "Electron-electron Interaction in Disordered Systems", A.J. Efros and M. Pollak Eds, Elsevier (1985).

[4] A.V. Andreev and A. Kamenev, Phys. Rev. Lett. 81, 3199 (1998).

[5] P.W. Brouwer, Y. Oreg and B.I. Halperin, Phys. Rev. B 60, R13977 (1999).

[6] H. U. Baranger, D. Ullmo and L. Glazman, Phys. Rev. B 61, R2425 (2000).

[7] T.A. Brody et. al., Rev. Mod. Phys. 53, 385 (1981); K.K. Mon and J.B. French, Ann. Phys. 95, 90 (1975).

[8] J. B. French and S. S. M. Wong, Phys. Lett. 33B, 447, (1970); 35B, 5 (1971); O. Bohigas and J. Flores, Phys. Lett. 34B, 261, (1971); 35B, 383 (1971).

[9] V. V. Flambaum, F. M. Izrailev and G. Casati, Phys. Rev. E 54, 2136 (1996).

[10] S. Åberg, Phys. Rev. Lett. 64, 3119 (1990); Ph. Jacquod and D. L. Shepelyansky, Phys. Rev. Lett. 79, 1837, (1997).

[11] V. V. Flambaum et. al., Phys. Rev. A 50, 267 (1994).

[12] V. Zelevinsky et. al., Phys. Rep. 276, 85 (1996).

[13] Y. Alhassid, Ph. Jacquod and A. Wobst, to appear in Phys. Rev. B, Rapid Comm.

[14] Due to SRS, states in the tail of each block's MBDOS have $\sigma=\left|\sigma_{z}\right|$.

[15] D. Weinmann, W. Häusler and B. Kramer, Phys. Rev. Lett. 74, 984 (1995). 